\title{
Estudo da demanda de saúde mental em Centro de Saúde da Família em Caucaia, Ceará, Brasil
}

\author{
Study of the mental health demand of a Family Health Center in Caucaia, Ceará, Brazil \\ Estudio de la demanda de salud mental en Centro de Salud Familiar en Caucaia, Ceará, Brasil
}

André Luís Bezerra Tavares. Universidade Federal do Ceará (UFC). andrelbtavares@yahoo.com.br (Autor correspondente)

Adriano Rodrigues de Souza. Universidade de Fortaleza (UNIFOR). adrianorsouza@gmail.com.br

Ricardo José Soares Pontes. Universidade Federal do Ceará (UFC). rjpontes@fortalnet.com.br

\section{Resumo}

Objetivo: Identificar a taxa de atendimentos a usuários com queixas de saúde mental em Centro de Saúde da Família, em Caucaia, CE, Brasil, de maio de 2011 a dezembro de 2011. Métodos: Estudo transversal descritivo, de abordagem quantitativa. Para coleta de dados foram aplicados SRQ-20 e formulário estruturado (idade, sexo, classificação, diagnóstico, uso de medicação e acompanhamento em outros serviços de saúde). Os dados foram analisados através de números absolutos e percentuais. Resultados: Verificou-se taxa de atendimentos médicos relacionados à saúde mental de 19,48\% (579) entre os realizados (2.972). Desses, 19,5\% (113) apresentavam transtorno mental leve a moderado, 34,7\% (201), transtorno mental grave, e 43\% (249), sofrimento psíquico. Conclusões: Constatou-se alta prevalência de transtornos mentais e sofrimento psíquico no serviço de saúde pesquisado. Torna-se necessária a implementação de ações de saúde mental na Estratégia Saúde da Família, com articulações intersetoriais e que contribuam para facilitar o acesso de usuários ao serviço de saúde, possibilitando reabilitação psicossocial.

\section{Abstract}

Objective: To identify the number of assistances to users with mental health complaints among users of a Family Health Center in the municipality of Caucaia, state of Ceará, Brazil, from May to December 2011. Methods: A cross-sectional descriptive study with quantitative approach. For data collection, we applied the SRQ-20 and a structured form (age, gender, classification, diagnosis, medication and follow-up in other health services). Data were analyzed using percentages and absolute numbers. Results: It was observed a rate of $19.48 \%$ (579) of medical consultations related to mental health in a total of $(2,972)$. Of these, $19.5 \%$ (113) presented mild to moderate mental disorder, $34.7 \%$ (201) severe mental disorder, and $43 \%$ (249) psychological distress. Conclusions: We identified a high prevalence of mental disorders and psychological distress in the health service investigated. It is necessary to implement mental health services in the Family Health Strategy, with inter-sectorial coordination and support to the access of users, providing psychosocial rehabilitation.

\section{Resumen}

Objetivo: Identificar la tasa de atención a usuarios con problemas de salud mental en el Centro de Salud Familiar en Caucaia, Ceará, Brasil, de mayo a diciembre/2011. Métodos: Estudio transversal descriptivo, cuantitativo. Para la recolección de datos, se aplicaron el SRQ20 y formularios estructurados (edad, género, clasificación, diagnóstico, medicamentos y acompañamiento en otros servicios de salud). Los datos fueron analizados a través de números absolutos y porcentajes. Resultados: Hubo una tasa de consultas médicas relacionadas a salud mental de 19,48\% (579) entre la atención médica realizada (2.972). De éstos, 19,5\% (113) presentaban trastorno mental leve a moderado, 34,7\% (201) trastorno mental grave y $43 \%$ (249) sufrimiento psicológico. Conclusiones: Se constató una alta prevalencia de trastornos mentales y sufrimiento psíquico en el servicio de salud investigado. Se hace necesario implementar acciones de salud mental en la Estrategia de Salud Familiar, con coordinación intersectorial y que ayuden a facilitar el acceso de usuarios a los servicios de salud, posibilitando la rehabilitación psicosocial.

\section{Palavras-chave:}

Saúde Mental

Epidemiologia

Atenção Primária à Saúde
Keywords:

Mental Health Epidemiology Primary Health Care 


\section{Introdução}

De acordo com a Organização Mundial da Saúde $(\mathrm{OMS})^{1}$, saúde mental seria um completo estado de bem-estar, no qual o indivíduo tem consciência de seu potencial, consegue lidar com problemas do dia a dia, pode trabalhar produtivamente e encontra-se apto a contribuir com a comunidade. Contudo, diferenças culturais, julgamentos subjetivos e outras teorias afetam o modo como a saúde mental é definida ${ }^{1}$.

Três por cento da populaçáo necessita de cuidados contínuos em saúde mental (portadores de transtornos mentais graves e persistentes); $10 \%$ a $15 \%$ precisam de atendimento eventual (portadores de transtornos leves e moderados ou comuns); $80 \%$ apresentam algum sofrimento psíquico, com tendência à remissão espontânea; $6 \%$ a $8 \%$ da população usam de forma prejudicial álcool e/ou outras drogas².

Entretanto, não há consenso com relação a esses dados. A partir de estimativas de prevalência em estudo americano, as prevalências para a população brasileira seriam de $24,2 \%$ para transtorno mental, $6 \%$ para transtorno mental grave e 3,1\% para transtorno mental persistente ${ }^{3}$.

Ao se estudar a demanda de saúde mental, deve-se ressaltar a dificuldade de separá-la da saúde física, sendo os transtornos mentais uma das grandes motivações de parte dos usuários que procuram atendimento médico em ambulatórios clínicos gerais. Estudos americanos demonstraram que $40 \%$ a $60 \%$ da demanda de saúde mental estava sendo atendida na Atenção Primária por médicos generalistas, verificando-se que cerca de 38\% (em média 24\%) dos pacientes de ambulatório geral teriam transtorno mental ${ }^{4}$.

Essa alta prevalência tem sido confirmada em estudos brasileiros. Detectou-se prevalência média de $56 \%$ de transtorno mental comum em 714 usuários de unidades da Estratégia Saúde da Família (ESF) de Petrópolis, Rio de Janeiro, sendo que 33\% do total de usuários apresentavam transtornos mais graves 5 . Outro estudo encontrou prevalência de $24,95 \%$ de transtorno mental comum em unidade da ESF em São Paulo ${ }^{6}$.

Estudo sobre prevalência de transtornos psiquiátricos na comunidade realizado em três capitais brasileiras apontou predominância de casos psiquiátricos que variou de 19\% (São Paulo) a 34\% (Brasília e Porto Alegre). Os transtornos ansiosos foram os mais prevalentes (18\%) e o alcoolismo, presente em todos os locais pesquisados, situou-se em $8 \%$. Os quadros depressivos apresentaram grande variação, de menos de 3\% (São Paulo e Brasília) até 10\% (Porto Alegre) ${ }^{7}$. Na Tabela 1 destacam-se os principais estudos de prevalência de transtornos mentais em unidades de saúde da família (USF), realizados no país.

Diante desse contexto, propôs-se este estudo com o objetivo de identificar a taxa de atendimentos a usuários com queixas de saúde mental em um centro de saúde da família.

Tabela 1. Prevalência de transtornos mentais em unidades de atenção geral à saúde no Brasil.

\begin{tabular}{|c|c|c|c|c|}
\hline Autor & Ano & Local & Instrumento & Resultados \\
\hline \multirow{2}{*}{ Busnello et al. ${ }^{8}$} & \multirow{2}{*}{1983} & \multirow{2}{*}{ Centro de saúde } & SRQ-20 & TMC: $55 \%$ \\
\hline & & & Entrevista clínica & Casos confirmados: $48,5 \%$ \\
\hline Mari $^{9}$ & 1987 & $\begin{array}{c}\text { Centro de saúde/Ambulatório } \\
\text { geral de hospital }\end{array}$ & $\begin{array}{c}\mathrm{GHQ}-12(2 / 3) \\
\mathrm{CIS}\end{array}$ & TMM: $22 \%$ a $27 \%$ \\
\hline lacoponi ${ }^{10}$ & 1989 & Centro de saúde & SRQ-20 & TMC: $53 \%$ \\
\hline Villano ${ }^{11}$ & 1995 & em hospital universitário & CIDI & TMM: 38\% \\
\hline \multirow{2}{*}{ Fortes $^{5}$} & \multirow{2}{*}{2004} & \multirow{2}{*}{ Unidade de saúde da família } & $\mathrm{GHQ}-12$ & TMC: 56\% \\
\hline & & & CIDI & TMM: 33\% \\
\hline
\end{tabular}

Fonte: Adaptado de Fortes (2004)5. TMC (Transtornos mentais comuns), TMM (Transtornos mentais maiores), SRQ (Seft-reporting questionnaire), GHQ (General health questionnaire), CIS (Clinical interview schedule), CIDI (Composite international diagnostic interview). 


\section{Métodos}

Estudo transversal descritivo, de abordagem quantitativa. Para produção dos dados, utilizou-se formulário estruturado, que foi preenchido pelo pesquisador responsável durante atuação no Centro de Saúde da Família Maria Firmino Mendes, localizado em Caucaia, CE, entre maio de 2011 e dezembro de 2011. Esse município integra a Região Metropolitana de Fortaleza, CE, sua área é de $1.227 .895 \mathrm{~km}^{2}$ e, em 2010, tinha população de 325.441 habitantes ${ }^{12}$.

Caucaia aderiu ao pacto de gestão em 2006 e encontra-se em gestáo plena, pertencendo à macrorregião de Fortaleza. Divide-se em seis distritos sanitários, compostos por 46 unidades de saúde da família (USF), 74 equipes de saúde da família, 49 equipes de saúde bucal e seis equipes de núcleos de atenção à saúde da família (NASF). Na atenção secundária, é município polo, sendo referência para nove municípios da região da 2a Célula Regional de Saúde (CRES), dispondo de um hospital municipal com serviços de urgência, clínica médica, pediatria, traumato-ortopedia e cirurgia. Também conta com uma maternidade, que atua como referência para pré-natal de alto risco, cirurgia cesariana e urgência pediátrica ${ }^{13}$.

Na saúde mental, possui dois centros psicossociais: um CAPS AD (álcool e drogas) e um CAPS geral. No nível terciário realiza serviços de hemodiálise. Há, ainda, uma clínica de especialidades médicas e um centro de especialidades odontológicas, além de outros estabelecimentos de saúde conveniados para diagnóstico e tratamento ${ }^{13}$.

O CSF Maria Firmino Mendes localiza-se no centro do município, atende população de 4.726 pessoas cadastradas pelos sete agentes comunitários de saúde. Uma microárea ainda permanece sem agente de saúde. A equipe profissional conta com uma enfermeira, três auxiliares de enfermagem, uma dentista, uma auxiliar de consultório dentário, três agentes administrativas, um porteiro e uma auxiliar de serviços gerais, além do médico, que é o pesquisador responsável por este estudo. Uma equipe NASF atua na área, contando com uma fisioterapeuta, uma farmacêutica, uma terapeuta ocupacional, um educador físico, uma nutricionista e uma fonoaudióloga ${ }^{14}$.

Participaram da pesquisa os usuários atendidos pelo médico da instituição investigada no período, tendo sido avaliados 2.972 usuários que procuraram o ambulatório.

Para avaliar sofrimento psíquico dos usuários atendidos, foi utilizado o self report questionnaire, SRQ-20. O SRQ tornou-se instrumento amplamente utilizado para suspeição diagnóstica dos transtornos mentais comuns. Na versão brasileira, foram retiradas as quatro questôes referentes a distúrbios psicóticos, mantendo-se a versão com 20 itens. O SRQ foi traduzido para oito idiomas diferentes, sendo utilizado em vários países. Na década de 1990, o SRQ-20 destacou-se como instrumento de triagem de morbidade psíquica mais utilizado nos estudos brasileiros, apresentando sensibilidade em torno de $83 \%$ e especificidade de $80 \%$ e revelando-se, ainda, indicador eficaz de morbidade ${ }^{15}$.

Neste estudo, o questionário foi aplicado, de forma oral, pela agente administrativa do centro de saúde (estudante de Serviço Social) aos usuários a serem atendidos pelo médico da equipe no período estudado. Cada usuário recebeu uma pontuação, informada ao médico antes do início do atendimento. Usuários com pontuação superior a sete no SRQ-20 foram incluídos no formulário da pesquisa e avaliados de acordo com os critérios da Classificação Internacional de Doenças - 10a Revisão (CID-10). Os campos do formulário: iniciais, sexo, data de nascimento, classificação, medicação em uso, outros serviços em que é acompanhado (além do CSF) e diagnóstico desse usuário foram então preenchidos de acordo com os critérios da CID-10.

Foram considerados transtornos mentais graves e persistentes aqueles que resultam em incapacitação importante, tais como esquizofrenia, transtornos mentais orgânicos e transtorno bipolar. Transtornos mentais leves a moderados seriam os de recorrência comum, com alguma incapacitação e resposta satisfatória a tratamento fármaco e psicológico como depressão, ansiedade, pânico e transtorno obsessivo-compulsivo ${ }^{16}$.

Utilizou-se o termo transtornos mentais comuns para descrever quadros de sintomas múltiplos e inespecíficos, como irritabilidade, nervosismo, dores, fadiga, esquecimento, pouca concentração e memória, assim como manifestações que poderiam caracterizar-se como sintomas depressivos, ansiosos ou somatoformes. Entretanto, o continuum e a comorbidade entre essas três síndromes, assim como a indiferenciação entre alguns dos sintomas, motivou o enquadramento transtornos mentais comuns, isto é, indivíduos que não necessariamente preencheram os critérios diagnósticos da CID-105.

O estudo obedece aos preceitos da Resolução 196/96 do Conselho Nacional de Saúde do Ministério da Saúde do Brasil ${ }^{17}$ e obteve aprovação do Comitê de Ética em Pesquisa da Universidade Federal do Ceará (UFC), conforme protocolo 069/11.

As respostas dos formulários foram transcritas sob a forma de variáveis, de acordo com o Quadro 1, para planilha do programa Microsoft Excel e os dados foram analisados por meio de números absolutos e percentuais ${ }^{18}$. 
Quadro 1. Análise dos dados.

\begin{tabular}{|c|c|c|}
\hline Variável & Resposta & Observações \\
\hline Número do questionário & A partir de 001 & Conta o total de usuários com queixas de saúde mental \\
\hline Sexo & 1-Masculino; 2-Feminino; 3-Não respondeu & $\begin{array}{l}\text { Os que não responderem esta questão serão } \\
\text { desconsiderados }\end{array}$ \\
\hline Data de nascimento & $\begin{array}{l}\text { Calcular a idade em anos a partir da data de } \\
\text { nascimento }\end{array}$ & $\begin{array}{l}\text { Os que não responderem esta questão serão } \\
\text { desconsiderados }\end{array}$ \\
\hline Classificação da queixa de saúde mental & $\begin{array}{l}\text { 1-Transtorno mental leve a moderado; } \\
\text { 2-Grave e persistente; 3-Sofrimento psíquico } \\
\text { (quadros com tendência à remissão espontânea) }\end{array}$ & $\begin{array}{l}\text { Os que não responderem esta questão serão } \\
\text { desconsiderados }\end{array}$ \\
\hline Medicamentos utilizados pelo paciente & $\begin{array}{l}\text { 0-nenhuma; 1-antidepressivo; 2- antipsicótico; } \\
\text { 3-benzodiazepínico; 4-moderador do humor; } \\
\text { 5-prometazina/biperideno; 6-anticonvulsivante; } \\
\text { 7-outros }\end{array}$ & Pode-se admitir mais de uma resposta para esta questão \\
\hline $\begin{array}{l}\text { Compartilhado com outro serviço de } \\
\text { referência }\end{array}$ & $\begin{array}{l}\text { 1-caps ad; 2-caps geral; 3-neurologista; } \\
\text { 4-geriatra; 5-emergência/internamento; 6-pediatra; } \\
\text { 7-outros }\end{array}$ & Pode-se admitir mais de uma resposta para esta questão \\
\hline Diagnóstico em saúde mental & $\begin{array}{l}\text { 1-ansiedade; 2-depressão; 3-psicose; } \\
\text { 4-álcool/drogas; 5-epilepsia; 6- luto; 7-demência; } \\
\text { 8-causas orgânicas; 9- bipolar; 10-dependência } \\
\text { benzodiazepínica; 14-não foi possível identificar }\end{array}$ & Pode-se admitir mais de uma resposta para esta questão \\
\hline Total de atendimentos & Número total de atendimentos realizados pelo médico & \\
\hline Atendimentos de saúde mental & $\begin{array}{l}\text { Número total de atendimentos de saúde mental } \\
\text { realizados pelo médico e registrados na planilha }\end{array}$ & \\
\hline
\end{tabular}

\section{Resultados}

Entre os atendimentos realizados pelo médico da equipe no período (2.972), verificou-se taxa de 19,48\% (579) de atendimentos a usuários com queixas relacionadas à saúde mental, dos quais 31,6\% (183) eram do sexo masculino e 68,4\% (396), do feminino.

A média de idade foi 51,5 anos, variando de 1 ano a 87 anos; 19,5\% (113) apresentavam transtorno mental leve a moderado; 34,7\% (201), transtorno mental grave e persistente; e 43\% (249), sofrimento psíquico (Tabela 2).

Tabela 2. Perfil dos usuários do centro de saúde da família investigado em Caucaia, CE, Brasil, 2011.

\begin{tabular}{lcr}
\hline \multicolumn{1}{c}{ Dados } & $\mathbf{N}^{0}$ & $\%$ \\
\hline Sexo & & \\
Masculino & 183 & 31,6 \\
Feminino & 396 & 68,4 \\
Total & 579 & 100,0 \\
Classificação & & \\
Leve - moderado & 113 & 19,5 \\
Grave - persistente & 201 & 34,7 \\
Sofrimento psíquico & 249 & 43,0 \\
Álcool-drogas & 16 & 2,8 \\
Total & 579 & 100,0 \\
\hline
\end{tabular}


Os diagnósticos encontrados foram transtorno de personalidade $(0,2 \%-1)$; transtorno alimentar $(0,3 \%-2)$; transtornos náo orgânicos do sono $(0,2 \%$ - 1); fobias $(0,3 \%$ - 2); transtorno de ansiedade $(11,6 \%$ - 67); depressão (11,4\% - 66); transtorno afetivo bipolar (1\% - 6); transtorno psicótico (6,1\% - 35); uso problemático de álcool e/ou drogas (3,1\% - 18); transtorno mental orgânico $(22,3 \%$ - 129); sofrimento psíquico $(30,3 \%$ - 175); uso inadequado de benzodiazepínicos $(12,6 \%$ - 73$)$; e diagnóstico indefinido $(0,5 \%-3)$. A Figura 1 ilustra esses dados.

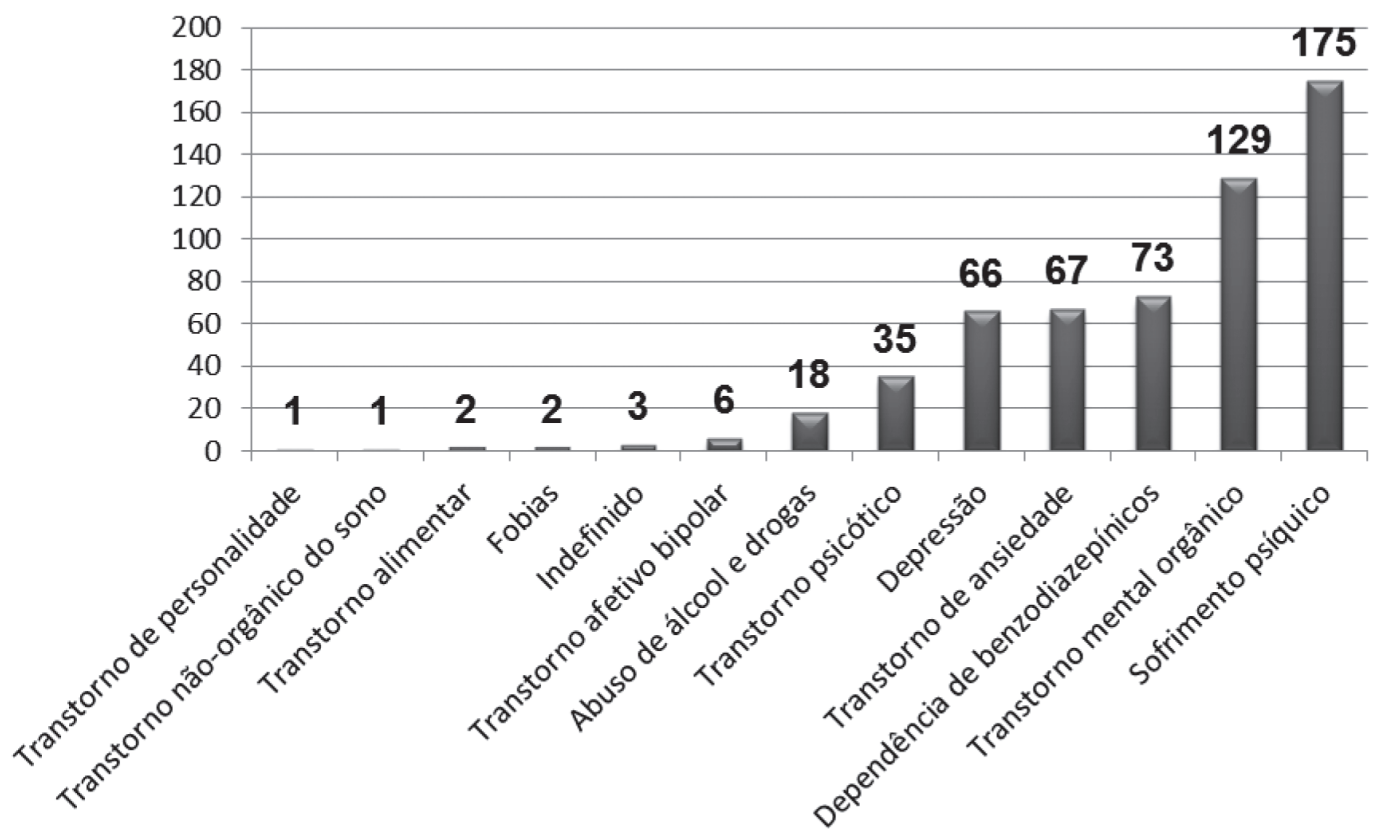

Figura 1. Distribuição dos principais diagnósticos identificados no estudo. Caucaia, CE, Brasil, 2011.

Parte dos usuários eram acompanhados pela Estratégia Saúde da Família (60,1\% - 348), outros (39,9\% - 231) eram compartilhados pelos centros de atenção psicossocial (CAPS) geral (15,5\% - 90), CAPS AD (2,8\% - 16), por neurologistas $(14,3 \%-83)$, geriatras $(6,4 \%-37)$, pediatras $(0,3 \%-2)$ ou foram encaminhados para emergência e/ou internamento $(0,5 \%-3)$.

Com relação ao uso de medicação, foram identificados 366 (61,2\%) indivíduos que faziam uso de medicaçáo psicotrópica, sendo as principais: antidepressivo (37,4\% - 137), benzodiazepínico (38,5\% - 141), antipsicótico (16,7\% - 61), anticonvulsivante $(22,4 \%$ - 82), anticolinérgico $(18,3 \%$ - 67), fitoterápico $(6,6 \%$ - 24) e/ou moderador do humor $(1,1 \%-4)$. Desses indivíduos, 71,31\% (261) usavam apenas um tipo de medicação; 19,12\% (70) usavam dois tipos; 8,5\% (31) usavam três tipos; e 1,1\% (4) usavam quatro tipos diferentes de medicação psicotrópica. Para 36,8\% (213) desses usuários não foi prescrita medicação psicotrópica.

\section{Discussão}

Dentre os atendimentos realizados pelo médico da equipe do centro de saúde no período analisado (2.972), a taxa de usuários com queixas de saúde mental encontrada foi de 19,48\% (579), o que confirma dados de estudo semelhante ${ }^{15}$. Entretanto, o fato de os sintomas apresentados pelos usuários com transtornos mentais comuns nem sempre se adequarem perfeitamente às bases categóricas dos diagnósticos psiquiátricos pode levar ao náo reconhecimento do transtorno por parte dos profissionais, que em geral enfocam a queixa somática imediata. Náo há consenso sobre a definição de transtorno mental comum. Pode-se, assim, tentar explicar a diferença encontrada entre a prevalência desses transtornos neste estudo (19,5\%) e a encontrada em outros estudos na literatura ${ }^{6,19}$. Outra causa poderia ser o delineamento metodológico deste estudo, que considerou, por exemplo, apenas os atendimentos direcionados ao médico da equipe, não considerando aqueles feitos pela enfermagem. 
A dificuldade de se reconhecer os transtornos mentais comuns apresenta como consequências, além da complexidade de acolhimento dessas manifestações de sofrimento, gastos com encaminhamentos e exames desnecessários, desdobramento de subdiagnósticos dessas manifestaçôes e prescrição abusiva de benzodiazepínicos ${ }^{6}$.

Observou-se maior prevalência de atendimentos de saúde mental entre mulheres $(68,4 \%)$, corroborando dados da literatura, uma vez que um dos achados mais persistentes foi que mulheres apresentaram prevalência de ansiedade e depressão duas a três vezes maior que homens ${ }^{20,21}$. Destaca-se a associação das desigualdades de gênero e o sofrimento ou comprometimento mental, mediado pela baixa autoestima das mulheres e seu menor controle sobre o meio ou contexto de vida ${ }^{22,23}$. Além disso, tanto a construção do menor valor dos afazeres das mulheres na sociedade quanto os desgastes e as percepçóes de pior saúde decorrentes dessas desigualdades de gênero fazem com que mulheres expressem mais facilmente seus sintomas, procurem mais pelos serviços de saúde que os homens e sejam efetivamente tratadas por profissionais de modo diverso ${ }^{23,24}$.

Entre as faixas etárias mais atendidas estavam os usuários com idade entre 41 e 70 anos, correspondendo a $60 \%$ dos casos. Analisando o ciclo de vida de pessoas nessa faixa, percebe-se que esse grupo etário corresponde em geral a casais na meia-idade e/ou famílias envelhecendo, o que pode gerar dificuldades adaptativas importantes. Essas constituem etapas em que os filhos estáo crescendo, muitas vezes deixando "o ninho". Começa-se a repensar a vida, a saúde, a morte, as perdas e o futuro financeiro ${ }^{25}$. Salienta-se que o delineamento deste estudo não permitiu conclusóes de causa e efeito, sendo essa apenas uma hipótese que merece maior investigação.

Ao se analisar os casos do ambulatório em estudo, nota-se que houve predomínio mais acentuado (34,7\%) de transtornos mentais graves e persistentes. Tal achado pode estar relacionado ao delineamento do estudo e ao fato de as pessoas com transtornos mais graves buscarem mais pelo serviço de saúde, ao passo que as portadores de transtornos mais leves buscam pelo serviço, na maioria das vezes, quando o quadro está se agravando e/ou cronificando. Além disso, esses usuários em geral são também acompanhados em outros serviços, usam medicação controlada e, assim, nos processos de triagem conseguem acesso ao atendimento médico mais facilmente. Os casos mais leves, geralmente, são resolvidos pela enfermagem ou não conseguem atendimento.

Em face ao contexto, destaca-se que os CAPS são serviços de saúde municipais e comunitários que oferecem atendimento diário e reinserção social às pessoas com transtornos mentais graves e persistentes, tentando adequar-se à realidade sociocultural de cada região ${ }^{26}$. Assim, para as pessoas com transtornos mentais graves e persistentes existe política de saúde mental eficaz em andamento no Brasil. Entretanto, para os transtornos menos graves não há política mais efetiva, levando esses usuários a sofrerem grandes entraves na busca de assistência ${ }^{3}$.

Salienta-se que os transtornos mais graves necessitam de acompanhamento mais complexo, que envolva a atençáo primária e secundária. Os demais podem ser em geral tratados na atenção primária e, em pequena proporção, por especialistas. Os casos de sofrimento psíquico, como luto, dificuldades de ajustamento, problemas afetivos, entre diversos outros, em geral têm tendência à remissão espontânea e necessitam mais de apoio do que de habilidades em saúde mental ${ }^{16}$.

Neste estudo registraram-se $2,8 \%$ de usuários com uso problemático de álcool e outras drogas. Prevalência distinta da referida pelo Ministério da Saúde, de cerca de $6 \%$ a $8 \%{ }^{2}$. Entretanto, os instrumentos utilizados neste estudo não são adequados para realizar tal diagnóstico.

No referente à prescrição de psicotrópicos, verificou-se que 63,2\% dos usuários foram medicados. Apesar de não haver dados suficientes na literatura sobre a medicalização do sofrimento sem a real indicação para o uso de psicotrópicos, indagou-se sobre o fato de muitos desses usuários não possuírem indicação formal para o uso desses medicamentos.

De acordo com a CID-10 são considerados transtornos mentais orgânicos aqueles cuja etiologia é uma doença ou lesão cerebral ou outro comprometimento que ocasiona a disfunção cerebral. Essa disfunção pode ser primária, como em doenças, lesôes e comprometimentos que afetam o cérebro de maneira direta e seletiva; ou secundária, como em doenças e transtornos sistêmicos que atacam o cérebro, bem como qualquer dos múltiplos órgãos ou sistemas envolvidos. São exemplos: demência, AVC, epilepsia, delirium, entre outros ${ }^{27}$.

Diante da alta prevalência de transtornos mentais orgânicos (129 - 22,3\%), percebeu-se grande número de usuários em acompanhamento compartilhado por profissionais da neurologia (14,3\%). Esse percentual foi aproximado daqueles acompanhados pelos CAPS (18\%) do município em que se localiza o centro de saúde investigado.

É inegável a importância da saúde mental no contexto da Estratégia Saúde da Família (ESF) para potencializar a qualidade de vida de usuários, famílias e comunidade. Assim, podem-se prevenir casos de violência e suicídios; pode-se esclarecer a 
população, combatendo o preconceito e a segregação social; evitar o abuso e a dependência de benzodiazepínicos; e colaborar para a construção da autonomia de familiares e usuários.

Pela otimização da capacidade resolutiva e da credibilidade da ESF evitam-se iatrogenias e constrói-se a desinstitucionalização, de acordo com a reforma da assistência psiquiátrica, com redução de gastos com internaçóes e demanda de centros de atenção psicossocial.

Parte considerável de profissionais de saúde ainda não capacitados para acolher e cuidar dessa demanda acaba estigmatizando usuários como poliqueixosos, psicossomáticos, pitiáticos. Distanciar esses usuários dessas categorias preconceituosas e perceber as necessidades de cuidado e acesso é um desafio para profissionais da atenção primária, assim como para seus processos de trabalho 5 .

As dificuldades que se interpõem ao acesso e cuidado das manifestaçôes de sofrimento que aparecem na APS, tanto por parte dos profissionais quanto da estruturação dos serviços, têm origem diversificada: pouca compreensão a respeito de códigos culturais diferentes para expressar o sofrimento; ausência de capacitaçôes adequadas para as equipes; serviços e profissionais ainda aprisionados no modelo individual e fragmentado de atendimento; prioridade das políticas de saúde mental para as clientelas consideradas graves; ausência de metodologias de cuidado e de acesso para a clientela com queixas difusas.

As principais limitaçóes deste estudo estão relacionadas ao fato de terem sido avaliados os atendimentos realizados pelo médico do CSF, o que certamente excluiu do estudo usuários com casos leves. $\mathrm{O}$ fato de a avaliação ter sido realizada em um único momento também leva a refletir sobre a exatidão dos diagnósticos psiquiátricos, dado que essas patologias necessitam de longo seguimento, passando por diversas mudanças em diagnósticos e tratamentos. $\mathrm{O}$ fato de náo terem sido avaliados mais fatores sociodemográficos também constituiu limitação deste estudo.

\section{Conclusões}

Este estudo confirma a alta prevalência de usuários com queixas relacionadas à saúde mental em serviços de atenção primária à saúde. Percebeu-se, assim, a necessidade de aprimorar o trabalho nessa área. A implementação de política pública, com implicações socioculturais da proposta da reforma da assistência psiquiátrica brasileira, necessita de articulação intersetorial e de açôes que contribuam para alterar as representaçôes sociais sobre sofrimento psíquico e os direitos dos usuários com transtornos mentais.

\section{Referências}

1. World Health Organization - WHO. Integrating mental health into primary care: a global perspective. Geneva: WHO/WONCA; 2008.

2. Brasil. Ministério da Saúde. Saúde mental e atenção básica. "O vínculo e o diálogo necessários": inclusão das ações de saúde mental na atenção básica. Brasília: Ministério da Saúde; 2003.

3. Campos GWS, Guerrero AVP, organizadores. Manual de práticas de atenção básica: saúde ampliada e compartilhada. São Paulo: Hucitec; 2008.

4. Organização Mundial da Saúde - OMS. Relatório sobre a saúde no mundo 2001 - Saúde mental: Nova concepção, nova esperança. Genebra: OMS; 2001.

5. Fortes S. Transtornos mentais comuns na atenção primária: suas formas de apresentação, perfil nosológico e fatores associados em unidades do programa saúde da família do município de Petrópolis, Rio de Janeiro, Brasil. [Tese]. Rio de Janeiro: Universidade Estadual do Rio de Janeiro; 2004.

6. Maragno L, Goldbaum M, Gianini RJ, Novaes HMD, César CLG. Prevalência de transtornos mentais comuns em populações atendidas pelo Programa Saúde da Família (Qualis) no município de São Paulo, Brasil. Cad Saúde Pública. 2006; 22(8): 1639-48. http://dx.doi.org/10.1590/S0102$311 \times 2006000800012$

7. Mello MF, Mello AAF, Kohn R, organizadores. Epidemiologia da Saúde Mental no Brasil. Porto Alegre: Ed. Artmed; 2007.

8. Busnello EL, Lima B, Bertolote JM. Aspectos interculturais de classificação e diagnóstico. J Bras Psiquiat. 1983; 4: $207-10$.

9. Mari JJ, Williams P. A validity study of a psychiatric screening questionnaire (SRQ-20) in primary care in the city of Sao Paulo. Br $J$ Psychiatry. 1986; 148: 23-6. http://dx.doi.org/10.1192/bjp.148.1.23

10. Iacoponi E. A Psiquiatria na Rede Primária de Saúde. In: Almeida OP, Dratcu L, Laranjeira R. Manual de Psiquiatria. Rio de Janeiro: Guanabara; 1996. p. 292-300. 
11. Fortes S, Villano LAB, Lopes CS. Perfil nosológico e prevalência de transtornos mentais comuns em pacientes atendidos em unidades do Programa de Saúde da Família (PSF) em Petrópolis, Rio de Janeiro. Rev Bras Psiquiatr. 2008; 30(1): 32-7. http://dx.doi.org/10.1590/S1516-44462006005000066

12. Instituto Brasileiro de Geografia e Estatística - IBGE. Cidades@ [Internet]. 2010. [citado em 2010 out 11]. Disponível em: http://www.ibge.gov.br/ cidadesat/

13. Caucaia. Secretaria Municipal de Saúde de Caucaia. Relatório Anual de Gestão. Caucaia: SMS; 2010

14. Tavares ALB, Souza AR, Pontes RJS. Mental health consultation profile in a family health center; 15th World Congress of Psychiatry; 2011, Buenos Aires. Buenos Aires: MCl; 2011. p. 505-505

15. Fonseca MLG, Guimarães MBL, Vasconcelos EM. Sofrimento difuso e transtornos mentais comuns: uma revisão bibliográfica. Rev APS. 2008; 11(3): 284-93.

16. Goldberg DG, Gournay KJM. The general practitioner, the psychiatrist and the burden of mental health care. London: Institute of Psychiatry; 1997. Maudsley Discussion Paper, n. 1.

17. Brasil. Conselho Nacional de Saúde. Resolução n. 196, de 10 de outubro de 1996. Dispõe sobre diretrizes e normas regulamentadoras de pesquisas envolvendo seres humanos. Bioética. 1996; 4(2 Supl): 15-25.

18. Pagano M, Gauvreau K. Princípios de Bioestatística. São Paulo: Cengage Learning; 2004.

19. Menezes TT, Melo VJ. O pediatra e a percepção dos transtornos mentais na infância e adolescência. Adolesc Saude. $2010 ; 7(3)$ : 38-46.

20. Patel V, Araya R, de Lima M, Ludermir A, Todd C. Women, poverty and common mental disorders in four restructuring societies. Soc Sci Med. 1999; 49(11): 1461-71. http://dx.doi.org/10.1016/S0277-9536(99)00208-7

21. Ludemir AB., Lewis G. Links between Social Class and Common Mental Disorders in Northeast Brazil. Soc Psychiatry Psychitr Epidemiol 2001 ; $36: 101-107$. http://dx.doi.org/10.1007/s001270050297

22. Rosenfield S. The effects of women's employment: personal control and sex differences in mental health. J Health Soc Behav. 1989; 30(1): 77-91. http://dx.doi.org/10.2307/2136914

23. Jenkins R. Sex difference in minor psychiatric morbidity. Cambridge: Cambridge University Press; 1985.

24. Schraiber LB, Oliveira AFPL, Falcão MTC, Figueredo WS. Violência dói e não é direito: a violência contra a mulher, saúde e os direitos humanos. São Paulo: Editora UNESP; 2005.

25. Sartori ACR, Zilberman ML. Revisitando o conceito de síndrome do ninho vazio. Rev Psiquiatr Clín. 2009; 36(3): 112-21. http://dx.doi.org/10.1590/ S0101-60832009000300005

26. Andrade LOM, Barreto ICHC, Fonseca CD, Harzheim E. A Estratégia Saúde da Família. In: Duncan. Medicina Ambulatorial: Condutas de Atenção Primária Baseada em Evidências. 3. ed. Porto Alegre: Artmed; 2006. cap. 7, p. 88-99.

27. Organização Mundial da Saúde - OMS. Classificação de Transtornos Mentais e de comportamento da CID - 10: Descrições Clínicas e Diretrizes Diagnósticas. Porto Alegre: Editora Artmed; 1998 